BULL. AUSTRAL. MATH. SOC.

MOS $05 A 05$

VOL. $5(1971), 197-202$.

\title{
A finite set covering theorem
}

\section{Alan Brace and D.E. Daykin}

Let $n, s, t$ be integers with $s>t>1$ and $n>(t+2) 2^{s-t-1}$. We prove that if $n$ subsets of a set $S$ with $s$ elements have union $S$ then some $t$ of them have union $S$. The result is best possible.

\section{Introduction}

Small letters denote non-negative integers and large letters denote sets. In particular 0 is the empty set, and $[i, j]$ denotes the set $\{i, i+1, i+2, \ldots, j\}$. Suppose that $X_{1}, X_{2}, \ldots, X_{n}$ are subsets of the set $S=[1, s]$ which cover (have union) $S$. We are here concerned with determining the smallest number of $X_{i}$ which will cover $S$. Of course $s$ of the $X_{i}$ will cover $S$, just take a suitable $X_{i}$ for each element of $S$. However can we be sure that $t$ of the $X_{i}$ will cover $S$ if $t<s$ ? At the other extreme we could have all the $2^{s}-1$ proper subsets of $S$ with no $t=1$ of them equal to $s$. So we assume $s>t>1$, and then an important example is

$$
E=\{X ; X=P \cup Q, P \subset[1, t+1],|P| \leq 1, Q \subset S \backslash P\} .
$$

Since no set $X$ in $E$ contains two elements of $[1, t+1]$ it is clear that no $t$ sets of $E$ have union $S$, and the number $e$ of sets in $E$ is

$$
e=e(s, t)=(t+2) 2^{s-t-1}
$$

We can now state our

Received 31 March 1971. 
THEOREM. Let $n, s, t$ be integers with $s>t>1$ and let $N=\left\{X_{1}, X_{2}, \ldots, X_{n}\right\}$ be $n$ different subsets $X_{i}$ of $s=[1, s]$ with zonion $S$. Suppose also that no $t$ of the $x_{i}$ have union $S$. Then

(i) $n \leq e$, and

(ii) if $3 \leq t$ and $n=e$ we can obtain $N$ from $E$ by permuting the elements of $S$.

When $t=2$ we can attain the value $e$ in many ways beside $E$, for instance

$$
F=\{X ; X=[1] \text { or } X \subset[2, s], X \neq[2, s]\}
$$

or

$$
G=\{X ; X=[1] \cup Y \text { or } X=[2] \cup Y \text { or } X \subset[3, s], Y \subset[4, s]\}
$$

and so on. If in an application of the theorem one knew that the $X_{i}$ have non-empty intersection $T$ one could improve the result by restriction to $S \backslash T$.

\section{Proof of (i)}

Without loss of generality we strengthen the hypothesis of the theorem by assuming that $n$ is as large as possible. This implies that if $X$ is in $N$ then all subsets of $X$ are in $N$. When $t=2$ we can't have a subset $X$ of $S$ and its complement both in $N$ so $n \leq \frac{1}{2} 2^{s}=e(s, 2)$ and (i) holds. When $t=s-1$ no set $X$ of $N$ can have more than one element, so $n \leq s+1=e(s, s-1)$ and again $(i)$ holds. We now use double induction on $s, t$. We suppose $3 \leq t \leq s-2$ and that ( $i$ ) holds in the two cases $s-1, t$ and $s-1, t-1$. Then we deduce that ( $i$ ) holds for the case $s, t$. Clearly some set of $N$ has more than one element so we assume $[1,2]$ is contained in some set in $N$.

To define a partition of $N$, for brevity we write 1,2 and $1 \cup 2$ for the sets [1], [2] and [1,2], and put

$$
B=\{X ; X \cup 1 \cup 2 \notin N, X \cup 1 \backslash 2 \in N, X \cup 2 \backslash 1 \in N\} \text {. }
$$

Then the partition is

$$
N=A \cup B_{0} \cup B_{1} \cup B_{2} \cup C_{1} \cup C_{2} \cup D
$$


where

$$
\begin{aligned}
A & =\{X ; X \cup 1 \cup 2 \in N\} \\
B_{0} & =\{X ; X \in B, 1 \notin X, 2 \notin X\} \\
B_{1} & =\{X ; X \in B, 1 \in X\} \\
B_{2} & =\{X ; X \in B, 2 \in X\} \\
C_{1} & =\{X ; X \cup 1 \backslash 2 \in N, X \cup 2 \backslash 1 \notin N\} \\
C_{2} & =\{X ; X \cup 1 \backslash 2 \notin N, X \cup 2 \backslash 1 \in N\}
\end{aligned}
$$

and

$$
D=\{X ; X \cup 1 \backslash 2 \notin N, X \cup 2 \backslash 1 \notin N\} \text {. }
$$

Let $a, b_{0}, b_{1}, \ldots$ denote the number of elements in the sets

$A, B_{0}, B_{1}, \ldots$ respectively, even though some of these sets may be empty. If $X$ is in $C_{1}$ then all subsets of $X$ are in $N$ so

$$
X \cup 1, X \backslash 1 \in C_{1} \text { for all } X \in C_{1} \text {. }
$$

By similar reasoning we see that

$$
B_{1}=\left\{Y ; Y=X \cup 1, X \in B_{0}\right\} \text { and } B_{2}=\left\{Y ; Y=X \cup 2, X \in B_{0}\right\}
$$

and hence $b_{0}=b_{1}=b_{2}$.

Case 1. $b_{0} \leq d$. In this case we put

$$
\begin{aligned}
& C_{2}^{\prime}=\left\{X ; X \in C_{2}, 2 \notin X\right\} \cup\left\{Y ; Y=X \cup 1 \backslash 2, X \in C_{2}, 2 \in X\right\} \\
& D^{\prime}=\{Y ; Y=X \cup 1, X \in D\}
\end{aligned}
$$

and

$$
N^{\prime}=A \cup B_{0} \cup B_{1} \cup D^{\prime} \cup C_{1} \cup C_{2}^{\prime} \cup D \text {. }
$$

We have chosen $N^{\prime}$ in such a way that, like in (1), we have

$$
X \cup 1, X \backslash 1 \in N^{\prime} \text { for all } X \in N^{\prime} \text {. }
$$

Since $c_{2}^{\prime}=c_{2}$ and $d^{\prime}=d$ the number of sets in $N^{\prime}$ is $n^{\prime}=n-b_{0}+d$. Roughly speaking the sets of $N$ and $N^{\prime}$ differ only with respect to the elements 1 and 2 . As $[1,2]$ is in $A$ it is clear that $N^{\prime}$ covers $S$.

Since (2) holds we can let $Y_{1}, Y_{2}, \ldots, Y_{\frac{1}{2}} n^{\prime}$ be the sets of the form 
$\left\{Y ; Y \in N^{\prime}, 1 \notin Y\right\}$. These sets cover $[2, s]$ but we, claim that no $t$ of them do so. For suppose $y_{1}, Y_{2}, \ldots, y_{t}$ cover $[2, \varepsilon]$. Then the element 2 is in one of the sets, $Y_{1}$ say, and so $y_{1}$ is in $A$, because 2 is only in the sets $A$ of $N^{\prime}$. Moreover $y_{1} \cup 1 \cup 2$ is also in $A$. For $2 \leq k \leq t$ there is a set $x_{i_{k}}$ of $N$ which differs from $y_{k}$ only with respect to the elements 1 and 2 , so in $N$ we have

$$
S=\left\{y_{1} \cup 1 \cup 2\right\} \cup x_{i_{2}} \cup x_{i_{3}} \cup \ldots \cup x_{i_{t}},
$$

a contradiction. By our induction hypothesis $\frac{1}{2} n^{\prime} \leq e(s-1, t)$, so

$$
n \leq n-b_{0}+d=n^{\prime} \leq 2 e(s-1, t)=e(s, t) \text {, }
$$

and (i) holds in this case.

Case 2. $b_{0}>d$. We show that this case never arises. We put

$$
\begin{aligned}
B_{3} & =\left\{Y ; Y=X \cup 1 \cup 2, X \in B_{0}\right\} \\
M & =A \cup B_{0} \cup B_{1} \cup B_{2} \cup C_{1} \cup C_{2} \cup B_{3} \\
L & =\{[i] ; i \notin M, i \in S\}
\end{aligned}
$$

and

$$
N^{\prime \prime}=L \cup M
$$

If the sets in $M$ cover $S$ then $L$ is empty, but if $L$ is not empty then $L \subset D$ because $N$ covers $S$. Also $n^{\prime \prime}=n+b_{0}-d+2>n$, so because the $X_{i}$ were chosen with $n$ as large as possible, there are $t$ sets $z_{1}, z_{2}, \ldots, z_{t}$ in $N^{\prime \prime}$ which cover $S$. If $L \neq 0$, every set [i] in $L$ is a $z_{k}$, for otherwise the element $i$ would not be covered.

We claim that no $z_{k}$ is in $A$. For otherwise for $1 \leq k \leq t$ we act as follows:

(a) if $z_{k} \in A$ let $X_{i_{k}}=z_{k} \cup 1 \cup 2 \in A$

(B) if $z_{k} \in B_{3}$ let $X_{i_{k}}=z_{k} \backslash(1 \cup 2) \in B_{0}$, and

$(\gamma)$ in all other cases let $X_{i_{k}}=z_{k}$. 
Then we have the contradiction that $x_{i_{1}}, x_{i_{2}}, \ldots, x_{i_{t}}$ cover $s$ in $N$. Next we claim that no two of the $z_{k}$ lie in

$$
H=B_{0} \cup B_{1} \cup B_{2} \cup B_{3} \cup C_{1} \cup C_{2} \text {. }
$$

Elements 1 and 2 must be covered by sets in $H$ because they are not covered by sets in $L$. So suppose $Z_{1}, Z_{2}$ are in $H$ and cover 1 and 2 . If $Z_{1}, Z_{2} \notin\left(B_{0} \cup B_{3}\right)$ they are in $N$ and we let $X_{i_{1}}, X_{i_{2}}$ be them. If $Z_{1}, Z_{2} \in\left(B_{0} \cup B_{3}\right)$ we put $X_{i_{1}}=z_{1} \cup 1 \backslash 2 \in B_{1} \subset N$ and $X_{i_{2}}=Z_{2} \cup 2 \backslash 1 \in B_{2} \subset N$. Finally if $Z_{1}$ is not in $B_{0} \cup B_{3}$ but $Z_{2}$ is in, we put $x_{i_{1}}=z_{1} \in N$ and $x_{i_{2}}=\left(z_{2} \backslash[1,2]\right) \cup j \in N$, where $j$ is that one of the elements 1,2 which is not in $z_{1}$. Then for $3 \leq k \leq t$ we act as in $(\beta)$ and $(\gamma)$ above to obtain $t$ sets in $N$ which cover $S$, $a$ contradiction.

Thus we conclude that $Z_{1}, Z_{2}, \ldots, Z_{t}$ consist of one set in $B_{3}$ and $t-1$ sets in $L$, so $z=t-1$. Without loss of generality assume these $t-1$ sets to be $[s-t+2],[s-t+3], \ldots,[s]$. We now observe that firstly, no set in $N$ contains more than one element of $[s-t+2, s]$, and secondly, no set in $N$ contains 1 or 2 together with an element of $[s-t+2, s]$. Otherwise we easily get $t$ sets of $N$ which cover $S$. Hence sets in $N$ containing the element $s$ must be of the form $W \cup s$ with $W \subset[3, s-t+1]$. The set $[3, s-t+1] \cup s$ itself cannot be in $N$ or again we would get $t$ sets of $N$ covering $S$.

It now follows that the element $s$ is in less than $2^{s-t-1}$ sets of $N$. No $t-1$ of the remaining sets cover $[1, s-1]$ so the number of these, by our induction hypothesis, is not greater than $e(s-1, t-1)$. Therefore $n<2^{s-t-1}+e(s-1, t-1)=e(s, t)$ and this is fewer sets than we get with example $E$, contradicting our assumption that $n$ was maximal. Thus this case is impossible, and (i) holds by induction.

\section{Proof of (ii)}

If $t=s-1$ then $N$ has no set with 2 elements, so $N$ is $E$, and (ii) holds in this case. We now use induction on $s$. In Section 2 
we showed that no $t$ of the sets $V=\left\{y_{1}, Y_{2}, \ldots, y_{\frac{1}{2} n^{\prime}}\right\}$ cover $[2, s]$, and we now have $n^{\prime}=e$. Thus by our induction hypothesis $V$ is of the same form as example $E$, and it is important to know whether or not the element 2 is in the set corresponding to $P$. Before discussing the cases we observe that if $2<i<j \leq s$ and no set in $V$ contains both $i$ and $j$ then no set in $N$ contains both $i$ and $j$.

Case 1. By permuting $[3, s]$ in $S$ we get

$$
V=\{Y ; Y=P \cup Q, P \subset[s-t, s],|P| \leq 1, Q \subset[2, s] \backslash P\}, 2 \notin P .
$$

Then after the permutation, no set in $N$ contains two elements of $[s-t, s]$, and since $N$ has $e$ sets, we must have

$$
N=\{X ; X=P \cup Q, P \subset[s-t, s],|P| \leq 1, Q \subset S \backslash P\} .
$$

Case 2. By permuting $[3, s]$ in $S$ we get

$$
V=\{Y ; Y=P \cup Q, P \subset[2, t+2],|P| \leq 1, Q \subset[2, s] \backslash P\}, 2 \in P .
$$

Consider any two elements of $[3, t+2]$, say 3 and 4 . Now for $5 \leq k \leq t+2$ there is a set $X_{k}$, say, in $N$ which contains the set $k \cup[t+3, s]$ of $V$. We claim that there are not two sets $X_{3}, X_{4}$, say, in $N$ with $1,3 \in X_{3}$ and $2,4 \in X_{4}$. Otherwise the $t$ sets $x_{3}, x_{4}, \ldots, x_{t+2}$ cover $S$ in $N$. Hence, because the elements 3,4 were chosen arbitrarily, either

$$
N=\{X ; X=P \cup Q, P \subset[1] \cup[3, t+2],|P| \leq 1, Q \subset S \backslash P\}
$$

or

$$
N=\{X ; X=P \cup Q, P \subset[2, t+2],|P| \leq 1, Q \subset S \backslash P\},
$$

and the theorem follows inductively.

University of Western Australia, Nedlands, Western Australia;

University of Reading,

Reading, England. 